\title{
Micro Systems Technology
}

\author{
Jan H.J. Fluitman \\ MESA Research Institute, University of Twente, \\ P.O. Box 217, 7500AE Enschede, The Netherlands
}

\begin{abstract}
The emerging field of Micro Systems Technology is described. Micro Systems Technology can be seen as the meeting of disciplines, a product of convergence along different lines. Apart from the traditional and ever developing line of "classical" precision engineering, there is a line along micro electronics, micro sensors and actuators. This is the line we focus on in this contribution. The third line worth mentioning is the one along the upcoming field of molecular engineering. The main purpose of this paper is to show the wealth of possibilities and consequently the need for "integral design" management.
\end{abstract}

\section{Introduction}

In this contribution we will treat the peripheral components of micro electronic systems. These are sensors and actuators. (Displays can be considered as a specific class of actuators.) Sensors and actuators have followed the way of IC's with respect to increasing functional densities, production technology and so on and there are continuous efforts to integrate these peripherals in "hard" and/or "soft" terms into the systems of interest.

This trend has started in the mid seventies when the impact of the microprocessor and other electronic functions became clear. We will spend some attention to the history of miniaturisation and the item of "smart" peripherals. But before we do so we will give a short introduction into micromachining of silicon, because this technology has been of great influence in the evolution of peripherals. So, in section 2 we will treat micromachining and after that we treat the new classes of sensors (section 3) and actuators (section 4). In section 5 we widen our scope and describe the increase of functional density in the past 25 years in general. This results in the emerging field of Micro System Technology to be described in the final section.

\section{Silicon Micromachining}

Before we start our treatment of micro sensors, actuators and systems, we will spend some words on micromachining of silicon. Micromachining as we use it here is a "sculpturing" technique using photo lithography, deposition, etching and bonding methods.

We restrict ourselves to silicon although micromachining can also be applied to quartz and GaAs-compounds. Silicon wafers are brittle. If they fall on a hard floor they break into sharp pieces because of the crystalline structure. Nevertheless the elastic properties of silicon are impressive. The yield strength is comparable to stainless steel and there is no region of ductility. The brittleness suggests that a silicon beam cannot bend without breaking. This is not true. A silicon beam of a few microns thickness and a length of a few hundreds of microns can be bent over 90 degrees without breaking and afterwards return to its original position. There are two kinds of micromachining, bulk and surface micromachining. Moreover bulk micromachining can be isotropic or anisotropic depending on the etchant. We will give some characteristic examples of devices made by either method.

Example 1. A bulk micromachined flow controller (1). Figure la gives a cross-section of a bulk micromachined flow controller. The machined wafer is sandwiched between two Pyrex wafers, which are anodically bonded to the silicon afterwards. Figure $1 \mathrm{~b}$ shows the steps that are used to shape

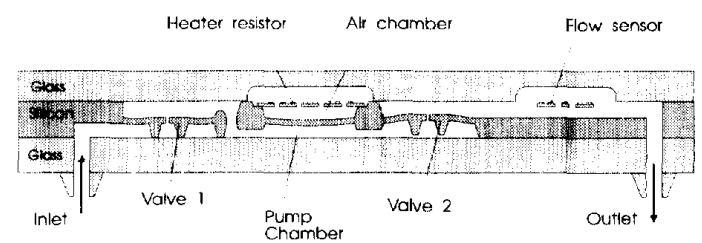

Fig. 1a. Cross section of integrated flow controller (1).

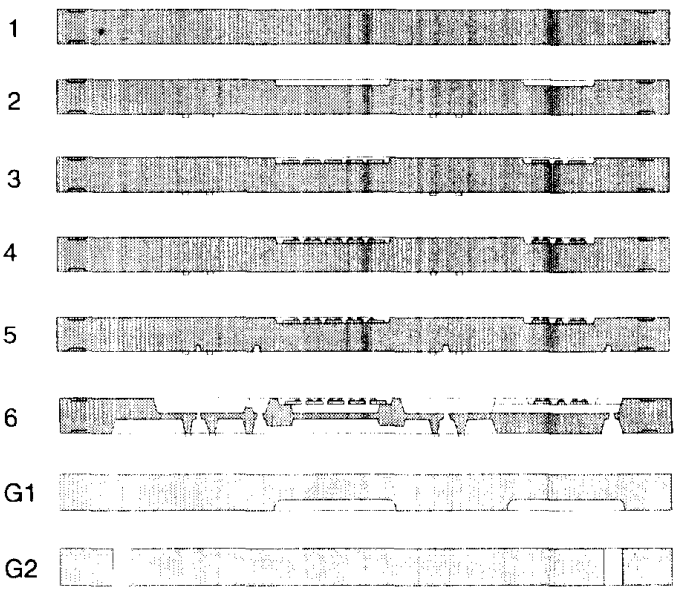

Fig. lb. Process sequence for the Si wafer and the two glass waters used in the integrated flow controller (1). 


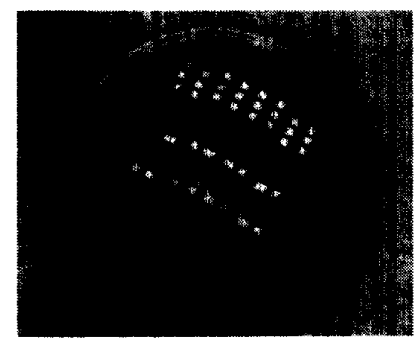

Fig. lc. Four micro pumps with micro flow sensors side by side on a three inch wafer.

the wafer. It can be seen that the principle of the method is to create windows to give access to the etchant. In this case an anisotropic etch is used in order to prevent under etching at the window sides. Figure 1c shows 4 pumps on a 3 inch wafer.

Bonding techniques, be it silicon to silicon, or other (possibly intermediate) materials are described in (2), we do not go into the details.

Example 2. A surface micromachined resonator sensor (3). Surface micromachining works with so-called sacrificial layers. These layers serve as a support for new layers. The sacrificial layers will be etched away afterwards by means of a selective etchant. Figure 2 a gives the procedure to produce a surface micromachined encapsulated resonator. Such a resonator has a resonance frequency depending on an exerted force in the length direction. This force can be introduced by bending the surface the resonator is built in. It then acts as a strain gauge. A prerequisite for a structure like this is that there is a residual stress in the free etched layer. Therefore the mechanical properties of the layers used must be well known in order to prevent buckling of the free etched layer. Figure 2c gives a SEM-photograph of the resulting sensor (with the "roof" torn away). The second structure is a non resonating dummy for compensation of parasitic influences.

A non-trivial part of bulk micromachining is the etch-stop mechanism. In order to make well defined membranes one must stop the etching at wish. There are several methods to do that (4), we will not treat them in detail here.

\section{Sensors}

A number of silicon based sensors were known to be feasible in the seventies, like the Hall-sensor, the membrane piezo resistive pressure sensor and the Ion Sensitive Field Effect Transistor (ISFET). After the breakthrough of the microprocessor two things became evident:

At first it was foreseen that the price/performance ratio of ICfunctions should decrease drastically, so that the existing peripherals were seen as constraints for a wide use of IC's.

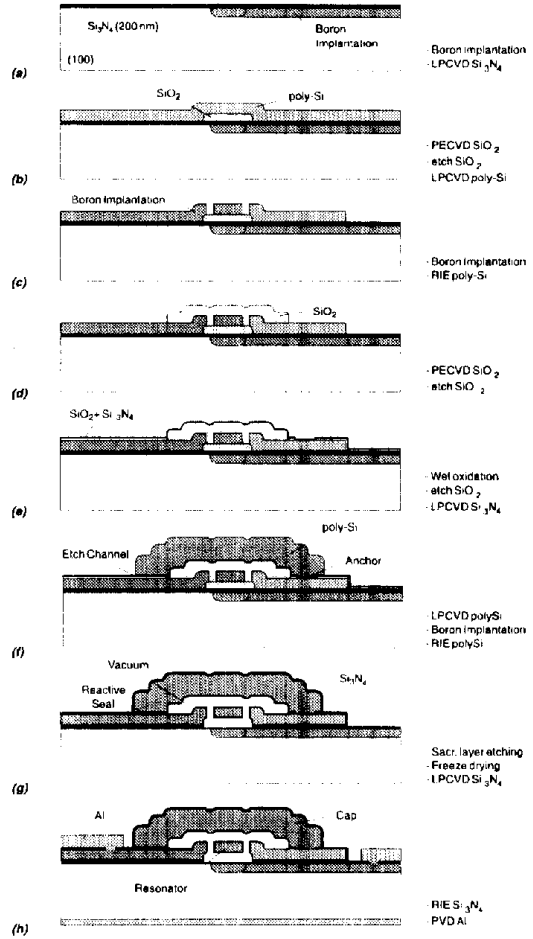

Fig. 2a Processing sequence for sealed resonators.

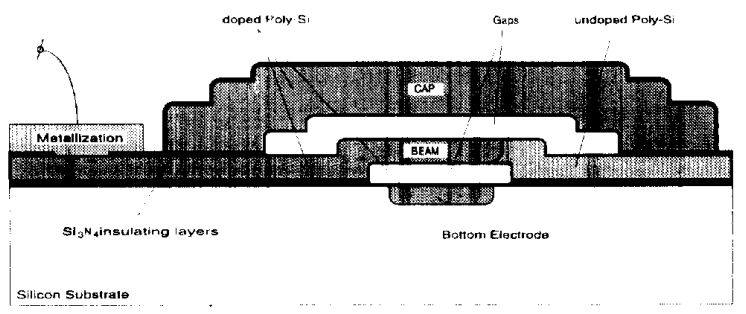

Fig. 2b. Cross-sectional view along the beam length (not drawn to scale)

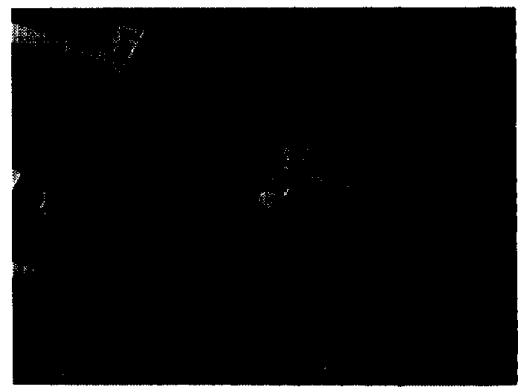

Fig. 2c. SEM-photograph of encapsulated beams (with the "roof" torn away from the lower left one). 
At second, with such a huge amount of $\mathrm{IC}$-function on a single chip there was room for one or more sensors on the chip. Or the reverse, which is a more realistic approach: if one or more sensor functions could be realized on the basis of silicon properties, then the non-optimal performance of silicon as a transducer material could easily be compensated for by some electronics right on the spot. Almost all transducer mechanisms (except piezo-electricity) are present in silicon or in silicon compatible material to some extent.

So there was a need and a solution based on the same progress of IC-technology. The need came forth out of the functional development of IC's, the possible solutions from the technological developments of IC-technology. This gave the search for a new class of "smart" sensors its great impetus. It was even thought that the "smart" sensors could be processed in a standard IC-line, so that the IC-producers saw a new branch of products for mass fabrication, the sensors, and at the same time a diminishing constraint for the application of their IC's.

This drive for smart sensors mentioned above can be seen as technology pushed, but there were market pulls as well. One of the most important ones was in the automotive world. In order to increase comfort and lower the burden on the environment there was a need for cheap reliable sensor systems.

Another pull concerns the ageing population in the world which requires cheaper and faster medical diagnosis and treatment systems. With respect to the economic use of raw materials and energy, and a reduction of harmful waste matter there was a pull from the side of process and product industry, while domestics forms an interesting market as well. Of course it is rather trivial that when you can sell more features for the same price there will be a market.

All market pulls mentioned above are still present and even stronger than ever. In the future the necessary legislation to prevent the world's decline will still put more pressure on the availability of these products.

How far are we with the smart sensor?

To answer this question it is good to realise that any sensor must "contact" the world. A sensor cannot be housed safely like an IC because it must have a look through the window. Some windows are relatively safe and others are not. To give an idea: if you want to measure a magnetic field you can shield the sensor completely. The only constraint is that you use no magnetic materials in the housing. On the other side a sensor to measure heavy metals in ground water must be exposed to a dirty substance and is nevertheless expected to send reliable information to the controls for a reasonable period of time.

And if you concentrate on a single parameter to sense, say pressure, than you might need a sensor to measure blood pressure, or in another application the pressure of a gas at 1000 degr. centigrade or in an highly erosive environment.

This means that sensors are in general no generic structures like IC's are. There are 50.000 different sensor products for about 100 parameters for sale in Europe at the moment (5). Smart sensors, in the original meaning, must operate in the temperature range of well functioning micro electronics and must be reasonably protectable which lays a burden on the encapsulation technology.

Nevertheless there is a large market for sensors in this area. Examples are the pressure, flow and acceleration sensors for use in the automotive world. And many of the high volume domestic and medical applications are working around room temperature.

So let us take an example from these applications, say the acceleration sensor (or deceleration sensor, if you think of triggering the inflation of the airbag, one of to day's Holy Grails).

An acceleration sensor consists of a seismic mass which can move as a consequence of elastic connections to a frame surrounding the mass. During acceleration or deceleration the mass moves and this can be detected either by piezo-resistive detectors (Fig. 3a) in the connecting supports or by means of electrostatic detectors with one electrode on the seismic mass and the other on the surrounding frame (Fig. 3b). There is quite a number of publications concerning these types of accelerometers made in silicon technology. Most of them concern bulk micromachined structures, a few of them surface micromachined structures.
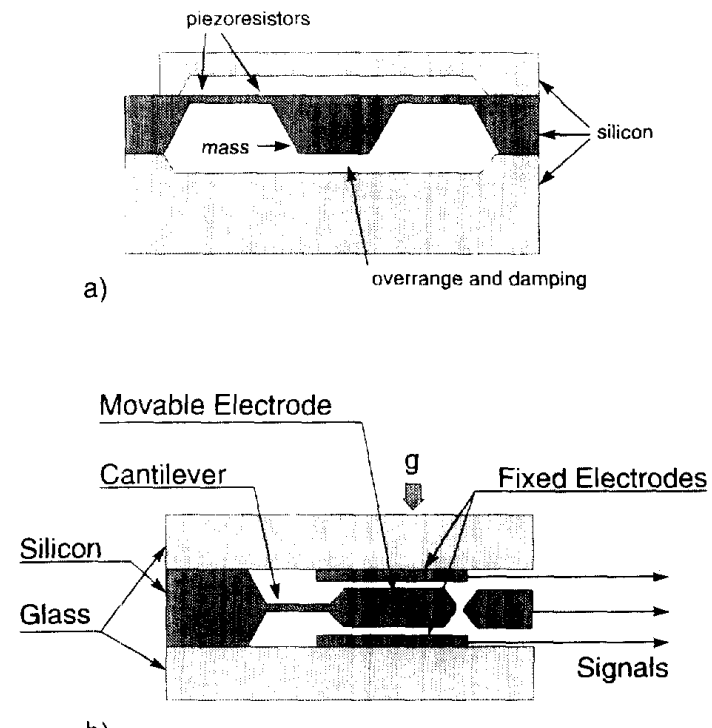

b)

Fig. 3. a) Piezoresistor-type accelerometer. b) Capacitance-type acceleromiter. 
There are efforts to integrate sensor and electronics in the same chip. A number of such efforts have the character of a demonstrator or are the result of a feasibility study $(6,7$, and $8)$. It is often unclear if the electronics is completely compatible with a standard IC-process, as can be found in an IC-foundry. The investigations are done with the help of an "in-house" IC-line, and minute steps in the IC-process which may deviate from a standard process (in order to get ahead of some "post-processing" steps), might be hidden in the report. This means, that the interest in the development of integrated accelerometers, is mostly in the hands of big companies that have access to such flexible facilities. This also means that it is hardly possible for Small and Medium Enterprises (SME) to join in. They need a design with the electronics prepared in an IC-foundry, followed by in house post processing, i.e. do the micromachining. One of the few groups working in this way is at the ETH-Zurich (Baltes, 9). To our knowledge no integrated accelerometers are designed that way.

Analog Devices announced a very elegant design, about three years ago (10). They are on the market now, figure 4 shows the scheme from the AD-data sheet concerning an air bag inflation accelerometer. The chip surface is about $3 \times 3 \mathrm{~mm}$., of which the sensor only takes about $7 \%$.

In the open literature there is some indication that renewed efforts are in the basic structure, not so much in integration.

It is unclear at the moment if the automotive industry chooses for integrated or hybrid accelerometers. It all depends on reliability, price and backwards compatibility. Of course it will take some time before mass products are introduced, which

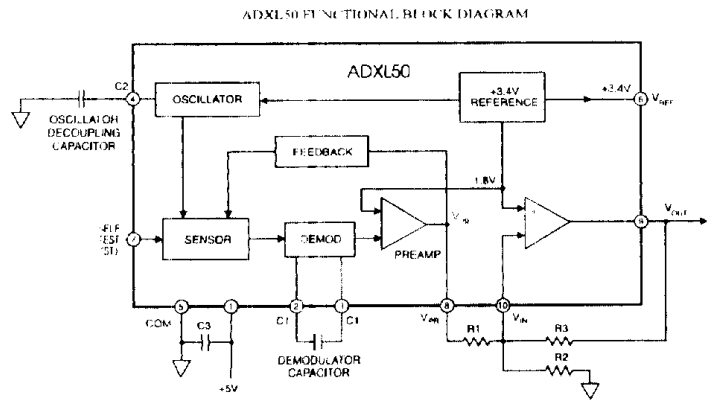

Fig. 4. Integrated accelerometer (data sheet Analog Devices).

have essential functions but no long history of succes. Note that an acceleration sensor must survive a 1 meter fall on a concrete floor $(1000 \mathrm{G})(12)$.

\section{Actuators}

An actuator acts, and needs some power to do that. This distinguishes the actuator from the sensor. Although sensing in principle has to do with power in one way or another, the possibility to miniaturize sensors is more obvious than actuators. An example is the magnetic recording head. When reading recorded information the head acts as a passive sensor, when writing information however, the write current must be high enough to create a head field that can produce a magnetization reversal in the recording layer.

Nevertheless in the eighties we have seen a breakthrough of micro actuators, based on micromachining techniques (12). As demonstrators one developed linear and rotating electrostatic micromotors. A lot of research has been done since that time and new fields of application have emerged. One often asks what you can do with actuators that small. My pertinent answer is: "Use your imagination!". But to be honest the question is mostly coming from a person who needs to solve the daily problems in his company and he or she might be a little bit frustrated by the glamour of these elegant tiny structures, which will probably function in the far future.

However there are some first applications. The accelerometer of Analog Devices shows the structure of the comb drive structures developed in Berkeley (13) and other places. So a device intended to be an actuator shows up in a sensor. This is true indeed, and now we come to a very important issue. The accelerometer has a self testing capacity. (In itself this is not new, there are other companies making self testing sensors.)

The important issue of self test is the presence of an actuator and the best solution you can have is a device that can act both as a sensor or an actuator. One and the same structure can be switched from one function to another.

It is not always possible to switch one and the same structure from a sensor to an actuator function.

Apart from self test, a reliable sensor of the modulator type should have an on board actuator in order to perform an in situ calibration. Modulator type sensors often show long term drift and you can not separate signal from drift without an additional actuator. An example is the ISFET which measures the $\mathrm{pH}$ of a liquid. An ISFET is a notorious drifter. In the case of the pH-sensing ISFET two electrodes, one on either side, are added (Fig. 5). If a current is sent through the electrodes the $\mathrm{pH}$ of the liquid is artificially changed (14). In this way coulometric acid-base titrations can be performed in nanoliter volumes within a few seconds. 


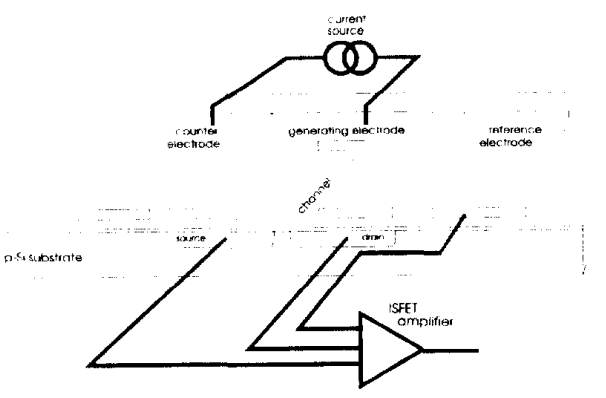

Fig. 5 Coulometric titration system.

Note, that in the mean time we have started to talk about systems. We have a sensor, an actuator and a piece of electronics to build a "smart" system. Reliable sensors need actuators. The same is true for an actuator like the micro pump of section 2. If there is no flow sensor on board you can hardly speak of a flow controller, because the pump may change is properties in the course of time. You can call this actuator drift

So the conclusion is, that since we can produce microactuators, we can produce more reliable sensors. This in itself is a very important application of micro actuators.

A separate proof of the capabilities of smart micro actuators is Texas Instrument's projection display (15), which is a surface micromachined array of $16 \times 16$ micrometer mirrors built on top of an electronic RAM to control the mirrors individually. We come back to this actuator in the next section.

But there are many more applications of micro systems. Before we go over to the subject of Micro System Technology (MST) however, we will present the development of systems in information technology during the last 25 years as an intermezzo. We will show the trend to miniaturization, which almost seems a law of nature. With this background we come along the question: "Why should all these things be small at all?". Is miniaturization just a trend or is it an evolution, that will take place anyhow?

\section{Miniaturization}

The peripheral devices of micro electronic structures have followed the trend to miniaturization of the electronics itself. The number of transistors on a memory chip have proven to grow faster then tenfold every five years.

Looking to the computer peripherals we see the same trend. External memories.

Hard disk drives have shrunken from the size of a frigidaire (1970) to the size of a matchbox (HP's Kittyhawk), with a much smaller price/performance ratio. The area bit density of a recording disk has grown with 4 or 5 decades the last 25 years. It is astonishing to see how magnetic recording breaks through barriers that where assumed impossible in the past. With the hot breath of alternatives in the neck, like magnetic bubble or CCD-memories, magnetic recording succeeded in beating the competitors. And we are curious to see how the battle with electronic flash memories will elapse. Possibly the precision engineered disk drives will turn into micromachined devices, who knows.

Displays.

Today's workhorse, the Cathode Ray Tube (CRT), is perfect for use on a fixed place, but for portable computer a flat panel display is required. This has lead to plasma discharge devices and Liquid Crystal Devices (LCD). For a colour display, pixels are needed with a typical feature size in the order of 100 micron. The pixels have to be controlled and the most efficient way to do this is to have some electronic functions at every position. One cannot think of large sheets of silicon as the basic substrate of an LCD-display. Therefore it is necessary to apply Silicon On Glass (SOG) technology. This means that $\alpha$-Si spots carrying the required electronics, e.g. a pair of diodes, are placed at any pixel position. For an overview of these techniques see Kuijk (16). The interesting thing occurring here is that transducers are not integrated on a silicon chip, but the reverse is true: electronics is integrated on a non-silicon transducer substrate.

Projection Displays.

Texas Instrument's new mirror array (15) is a marvellous example of miniaturization. On a RAM chip a mirror array is placed, using surface micromachining techniques after the RAM is produced. The mirrors (pixels) have a size of $16 \times 16$ microns and are fixed to torsional hinges in two opposite corners. The mirrors can be rotated to over 10 degrees to both sides. The rotation is forced by electrostatical attraction of the aluminiurn mirror and a ground electrode.

Communication channels.

For modern communication the invention of the glass fibre is of crucial importance. The basis of the communication hardware is the optical fibre with a core diameter of 1 micron or less. Compared to classical communication lines the amount of transported information has made a quantum leap while the medium is even much cheaper. For fast communication fast switching functions are necessary and this means that the use of integrated optic devices are necessary. The ideal is an opto-optic switch and research is done to develop integrated devices exploiting Non Linear Optical (NLO) materials.

Together with sensors and actuators, which we have treated in preceding sections, it is seen that the trend to miniaturization is almost a "natural" law for the complete set of peripherals. This is not a "trendy" trend. The requirement for miniaturization is a must for any progress in information technology. 


\section{Micro Systems Technology}

Why should systems be small at all?

The answer is hidden in the foregoing but we will explicitly add a number of additional reasons.

Small things are portable. The more features you can carry, the happier you are. It means that in doing your work, you are not pinned to a certain place. Work and workplace can remain connected, when you carry your workplace with you. Portability is also important in medical care. In that case you can carry diagnostic means with you and possibly the therapeutic means also. If the latter is not possible you can communicate with a physician or a hospital. Medical care must be cheaper in the future, because the world population is growing older and care is needed more and more. The problem cannot be solved by just an expansion of the medical care volume, because this leads to unpayable health insurance costs.

Diagnostic means coupled to therapeutical means may lead to artificial organs (e.g. an artificial pancreas) or to so called "smart pills". Again the answer of the question: "Why small?" is rather trivial.

In transport the number of micro systems must increase for better control of fuel costs, environmental load and so on. The systems must be small for the reason of payload. This is important for the automotive world, but also for aerospace applications.

The control of our environment has priority number one. In due course, legislation and inspection concerning the task of keeping our world liveable, presses the development of chemical analysis systems. There is a need for an incredible number of sensors, mostly (bio) chemical. In order to develop such sensors the need for on the spot calibration seems to be the only solution for reliable sensing. Drift and degradation, which are the greatest problems in making reliable sensors, can never be solved by "smartness" of the electronics. You need an actuator and thus a micro system. But apart from this, the quest for micro systems doing the sampling, the transport, the mixing, the reaction, the sensing, etc. are necessary to replace the existing bench equipment.

Minimal invasive surgery and drug delivery are two other functions that require miniaturization. In the case of drug delivery as well as in the case of micro chemical analysis there might be a need for disposable parts. Disposables should be small and cheap.

We can go on with this list of arguments but I think that these arguments are convincing and even point to urgency. See the appendix for a list of possible applications.

Micro Systems Technology is the working field to where all the new miniaturizing technologies can be oriented. And should be oriented. Of course we enter a field where all disciplines meet and it requires a new management of disciplines to uncover the treasures which are in the MSTfield in principle. This management is not only required to do the inventions, but it is also of great interest to define the infrastructure for industrial production of the new products. Production and producibility go hand in hand in design. Right at the start of a new design the disciplines including the fabrication technologies should come together and cooperate. Generally the production facilities for MST-products are very expensive and often dedicated. Time sharing of facilities especially for deposition of thin layers of different materials is highly problematic. But this is based on thinking in terms of the facilities as we know them now.

Of great importance is the development of new production facilities, which can do more, are more flexible, have a smaller volume and a lower price. So it is not only the products that miniaturize, the production facilities should go the same way, including clean rooms as fabrication units. If you calculate the ratio of the volume of the silicon present in an IC-line to the volume of the complete IC-line, including all of the air handling equipment, you arrive at crazy numbers. It is a great challenge to bring these numbers into a reasonable balance and to arrive at an acceptable ratio.

Up to now we have only spoken about so-called "sculpturing" technologies, in the line of the IC-technology. We have not mentioned the approach from the other side: making very small things bigger. Here I refer to the field of Supra Molecular Chemistry (SMC)(17). I am not a chemist, but I have had some looks inside this field where big molecules are designed or adapted, first by CAD-means and than in a real chemical process. The promises of this field of work are astonishing. And somewhere the "sculpturing" technologies and the SMC will meet and mix up.

The list of technological possibilities for fabrication and characterization is growing every year. With respect to fabrication we have not even mentioned the LIGAtechnology, Shape Memory Alloys, Soft Materials (e.g. Polymer Technology), Laser Induced 3D Lithography, Micro Electric Discharge Technology, Binary Optics, SIMOX technology, etc.

It is rather dazzling to keep control over all possibilities and press them into a single person's brains. Again we are confronted with the necessity to manage the disciplines and keep control over the sea of knowledge and possibilities.

\section{Conclusion}

The progress in MST has been impressive the last 25 years. If somebody in the sixties should have had the fantasy to predict, that individual atoms could be picked and placed at wish on a surface in 1990, he or she would have been placed in a madhouse. Today the equipment you need for doing this is for sale for reasonable prices and the greater part of the 
price covers the data processing equipment. The front end part of an Atomic Force Microscope is surprisingly simple.

And progress is going further: looking inside molecules and atoms. It seems that the thought experiments supporting Heisenberg's uncertainty relations of quantum mechanics can be turned into real experiments in due course.

This is what happened in the last 25 years.

A "freshly delivered" engineer looks forward to a 40 years career. I think it is really unimaginable what he or she is doing just before retirement and how the work is done at that time. So I just finish this contribution here, there is no more to say and too much to imagine.

Returning to the initial driver, the quest for "smart" sensors in the seventies, we must conclude that the development in technology and electronic means has proven to be so massive, that the perception on "smart" sensors of the seventies is overruled by the perception of "smart" systems. The meaning of "smart" is related to the systems function, not to its implementation. To be clear, it is of no use to talk about monolithic integration without a look to the developing alternatives, offered by hybridization techniques. It is even difficult to say whether a hybrid is a hybrid or a monolithic structure (think of silicon to silicon fusion bonding). The borderlines become rather fuzzy. Methods for hybridization, especially in the low (:- room) temperature range are under development.

There is a lot of work to do and this is related to the work in the field of custom integrated IC's. This work will be related to MST as a whole. How this work will proceed exactly is not known. The best one can do is to remain well informed and to create look out positions in companies and co-operate where and when the weight of such a position is too large for a single company.

Integration will go on in many senses and we will have to adapt to this process.

\section{Appendix}

\section{A choice of (possible) micro systems}

In the following list only systems are mentioned. So we mention no functions (e.g. pumps) or parts (e.g. micro valves). A breakdown of the system list into functions or parts will show overlap, but without any doubt leads to a large increase of the number of items. One must be aware of the fact that a system can be made out of a multitude of distributed microsystems. This is one of the strong points of MST.

Basic components:

Self testing/calibrating sensor systems, array type sensor systems, micro energy sources, wireless transport systems of energy and information, optical wave guide sensor systems, etc.

\section{Transport:}

Smart Engine Control, driver and passenger's comfort system, anti lock brake system (ABS), early warning system (e.g. oil, tyre pressure, driver half asleep), event recorder (micro "black box"), etc.

Medical:

Personal: Drug delivery systems, smart pills, artificial organs (e.g. artificial pancreas), organ control support (e.g. glaucoma), sensory organ support (hearing aid, cochlea implant), artificial "eyes", early warning systems (heart infarct), neuro-electronic connections, micro repair parts, disposables, revalidation systems, etc.

Hospital:

Catheter tip systems, micro surgery systems (e.g. micro motors), rnicro Total Analysis Systems (TAS) for medical analysis, etc.

\section{Environmental:}

Micro TAS for water (drinking water, ground water, river water, sea water, etc.) and for gases, stack monitoring, dust measurement, radioactivity measurement, etc.

\section{Production:}

Bearing controls, transport and conveyer integrity system, fine positioning and mounting systems, production systems for micro devices (including IC's), micro handling systems (e.g. tele-operation), micro TAS for chemical analysis, optical waveguide control systems, micro filters, micro cleaning systems, "in stream" process monitors, etc.

Safety and control:

Micro inspection units (e.g. in small tubes), surface inspection (micro cracks), smoke and gas signalling, identification systems, smart cards, quality control system of stored goods (liquids, bulk goods), etc.

Computer peripherals:

Projection systems (like TI's mirror system), recording systems, printing systems (e.g. nozzles), copiers (e.g. paper handling), electro-optic conversion systems, integrated optics systems, optical connectors and switches, micro-optic systems (e.g. CD), displays (e.g. active LCD-cells), etc.

Research and analysis.

Cell handling systems, nano-observation (including the dynamics), surface inspection, supra molecular instrumentation, probe microscopy, etc.

Agriculture/horticulture/animal farming.

Growth monitoring systems, plague warning systems, system for collection and distribution of pollen, nutrient and waste control, cattle status and identification systems.

Domestics:

Food quality control systems, (dish) washing control, cooking systems, micro dust cleaner, micro camcorder (parts) and other subsystems in the audio/video recording and display sector, etc.

Add:

Your own ideas. 


\section{References}

(1) T.S.J. Lammerink, M. Elwenspoek and J.H.J. Fluitman, "Integrated Micro-Liquid Dosing System", Proc. IEEE MEMS. Fort Lauderdale. USA. 1993, pp. 250-257.

(2) -W.P. Mazara, "Semiconducctor Wafer Bonding: An Overview"Ed U.Gosele e.a. The Electrochemical Society (Pennington NJ 1992p 82

-A Hanneborg, "Silicon Fusion Bonding for Fabrication of Sensors, Actuators and Microstructures", Proc. IEEE MEMS, Nara. Japan, 199/, p.92

(3) H.A.C.Tilmans, "Micromechanical Sensors using Encapsulated Builtin Resonant Strain Gauges", Ph.D.-Thesis, University of Twente, 1993.

(4) E.Peeters and B.Puers; "Development in Etch-stop Technique", Digest MME'93. Neuchatel, Switzerland 1993, pp. 35-50.

15) Harmer Associates, "European Research on Advanced Sensors", $A$ Report Sponsored by the Department of Trade and Industry under the Advanced Sensor Technology Transfer Programme, 1991.

(6) K.Murakami, "Development of Integrated Sensors for Automotive Applications", 18th Int Symp. Automotive Techn. and Automation. Vol.2, 1988. p.88076/1-17

(7) W.Riethmuller, W.Benecke, U.Schnakenberg and B.Wagner "Development of Commercial CMOS Process Based Technologies for the Fabrication of Smart Accelerometers", Digest Transducers 199/, pp. $416-419$

(8) J.C. Cole. "A New Sense Element Technology for Accelerometer Subsystems, Digest Transducers 1991, pp 93-96.

(9) H.Baltes, "CMOS as Sensor Technology", Sensors and Actuators $A$ Vol's $A 37-A 38.1993, \mathrm{pp} .51-56$.

(I0) F.Goudenough, "Airbags Boom when IC Accelerometers sees $50 \mathrm{G}^{\mathrm{n}}$, Electronic Design Aug 91. pp.45-56.

(1I) A Kölling, private communication.

(12) K.Gabriel, J. Jarvis and W.Trimmer (ed's), "Small Machines Large Opportunities: A Report on the Emerging Field of Microdynamics", Report of the NSF Workshop on Microelectromechanical Sysems Research, 1989.

(13) W.C.Tang. T.N. Nguyen and R.T. Howe, "Laterally driven Polisilicon Resonant Microsstructures", Proc. IEEE MEMS Salt Lake City, USA. 1989, pp.53-59.

(14) B. Van der Schoot, "Coulometric Sensors, Integration of a SensorActuator System", Ph.D.-Thesis. University of Twente, 1986

(15) J.M. Younse. "Mirrors on a chip", IEEE Spectrum. Nov. 1993, pp.2731 .

(16) K.E. Kuijk, "System Aspects of a Diode-Matrix Liquid-Crystal Television Display", Ph.D.-Thesis, University of Delft, 1993

(17) J-M. Lehn, "Supramolecular Chemistry - Molecules, Macro molecules and Molecular Functional Units", Nobel Prize Lecture (e.g. in Angew.Chemie 100, 1988 pp 91-116 (in German)). 